\title{
Volumetric Deformation Model for Motion Compensation in Radiotherapy
}

\author{
Kajetan Berlinger ${ }^{1}$, Michael Roth ${ }^{1}$, Jens Fisseler ${ }^{3}$, Otto Sauer ${ }^{2}$, \\ Achim Schweikard $^{3}$, and Lucia Vences ${ }^{2}$ \\ 1 Technische Universität München, Image Understanding and Knowledge-Based \\ Systems, Boltzmannstr. 3 D-85748 Garching bei München \\ berlinge@in.tum.de, \\ http://www9.in.tum.de/research/med \\ 2 Universitätsklinikum Würzburg, Radiology Department, \\ Josef-Schneider-Str. 11 D-97080 Würzburg \\ 3 Universität Lübeck, Institute for Robotics and Cognitive Systems, \\ Ratzeburger Allee 160 D-23538 Lübeck
}

\begin{abstract}
A new system is described that can track tumors moving due to respiratory motion, enabling specific and more effective irradiation of the tumor. This spares healthy tissue, and allows for higher doses to treat the tumor. To reach this goal a $3 \mathrm{D}$ motion picture of the lung of the specific patient has to be computed before treatment, because of the need to infer the respiratory state. This is done in a $4 \mathrm{D}$ registration step, matching 2D radiographs with the computed synthetic 3D scans. Morphing methods to calculate the deformation of the lung are described and evaluated.
\end{abstract}

\section{Introduction}

To apply radiosurgical methods to tumors in the chest and abdomen, it is necessary to take into account respiratory motion, which can move the tumor by more than $1 \mathrm{~cm}$. Without compensation, it is unavoidable to enlarge the target volume by a safety margin. As a consequence, healthy tissue is also affected. Therefore lower doses must be used to treat the tumor. In 1] we described a method using implanted fiducials to track the tumor. At the moment we are trying to achieve our goal without these fiducials, in order to avoid the encumbering operation for the patient and the medical staff. To solve this problem we need to compute a correlation between respiratory state and tumor position.

\section{Method}

Prior to treatment two CT scans are taken. One scan during inhalation, one during exhalation. From this data we then compute a series of intermediate synthetic CT scans. These are obtained by applying different interpolation methods, as described in section 3, to the CT scans taken at maximum inhalation and exhalation. This yields a sequence of 3D scans, showing the respiration process as a three-dimensional motion picture. 


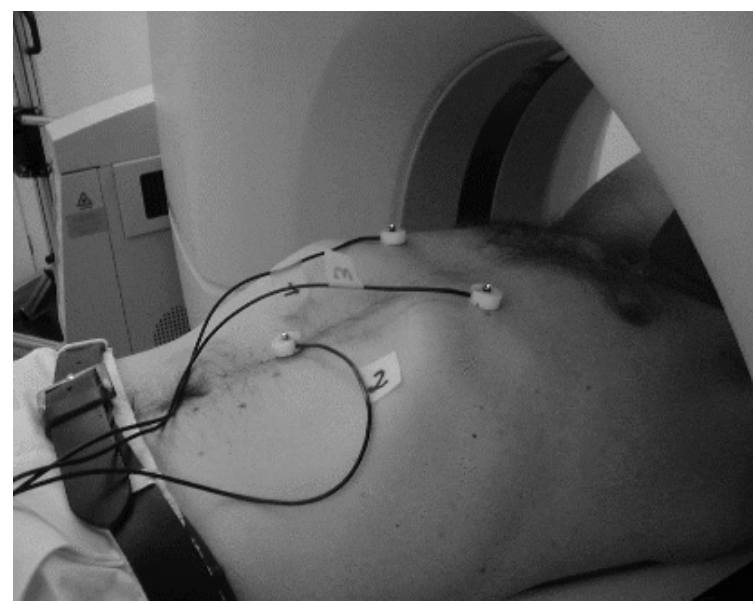

Fig. 1. The emitters, attached to the patient.

For each point in this time series, we compute a set of digitally reconstructed radiographs (DRR). A single DRR is computed by projecting a CT scan from a preselected angle using the known geometry of the imaging system. Thus a DRR can be regarded as a synthetic x-ray image, obtained by projecting through an existing CT volume. For each of the CT scans (synthetic or real), we compute a number of DRRs, each one for a preselected angle.

Before treatment, x-ray images are taken and compared to the set of DRRs we have created. (These x-ray images will be called live images). In these live images the tumor cannot be seen, but in the CT scans the target region is determined. After registration of the live image with the DRRs we know the best matching DRR in the series and therefore also the tumor position and the respiratory state, because of the stored association from a single DRR to its CT scan. Thus we acquire correlation from respiratory state to tumor position.

Instead of using pre-computed DRRs we are currently integrating a new method for online optimization using fast DRR calculation based on standard graphics hardware [2]. At the moment, registration time for a new live image is about 10 seconds for each stack.

This yields only intermittent information about the target location. At the time the comparison of all DRRs with the current live shot is completed, the target may already have moved. To solve this problem we use a sensor to report information on the current state of respiration in real time. This sensor is an infrared tracking system, with emitters attached to significant positions on the patient's chest and abdomen (figure 1). Kubo and Hill 7] compared various external sensors, like breath temperature sensor, strain gauge or spirometer, in matters of their applicability for respiratory gating. We use the infrared tracking method within our application, because of its high accuracy and speed as well as its stability under radiation exposure. 
The information of the sensor is correlated to the target location computed by the comparison between the live shot and the DRRs. Therefore, the live shot has a time stamp, and we can determine which reading of the real time sensor corresponded to this point in time. Repeating this time stamp synchronization, a complete correlation model can be obtained, which correlates target motion to the readings of the real time sensor (internal to the external motion), without internal fiducials.

The correlation allows the computation of the position of the target volume only. However the therapeutical beam has to reach the target volume, which is in moving, early enough. Both synchronization (gating) and active tracking have latencies due to physical limitations of the particular technique. Therefore predictive tracking uses the periodicity of the particular anatomical signal (respiration or pulsation), in order to compensate for this latency [1].

In figure 2 the setup of the system described above is shown.

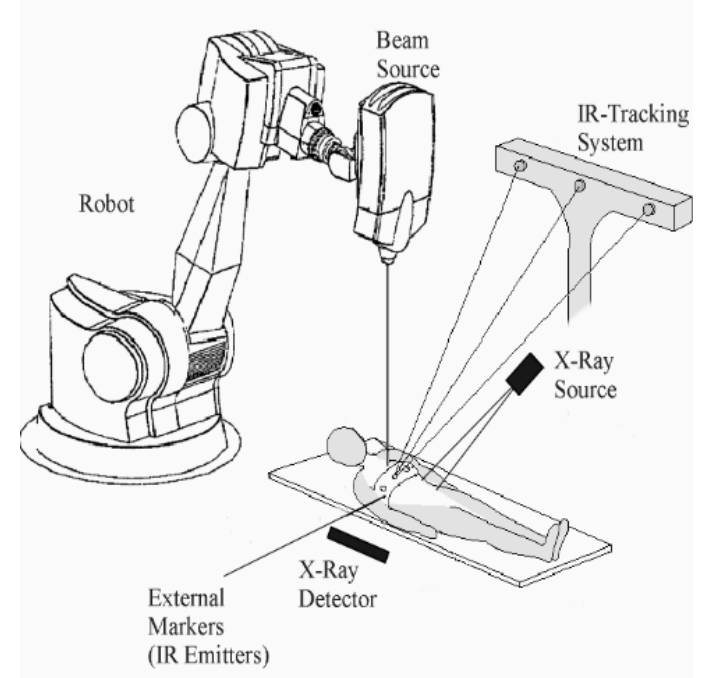

Fig. 2. The setup of our system, with an example of a linear accelerator, an infrared tracking camera and an x-ray device

\section{Deformation of the Lung}

Brock et al. 9 used finite element analysis to create a four-dimensional model of the liver. We considered two methods for the deformation of the lung, one based on tetrahedrization and the other on thin-plate splines. Both methods need as input: 
1. Two initial CT scans, taken during inhalation respectively exhalation (figure 3)

2. The number of desired interpolation steps (number of image stacks of the resulting $3 \mathrm{D}$ motion picture)

3. An arbitrary amount of pairs of corresponding control points

a)

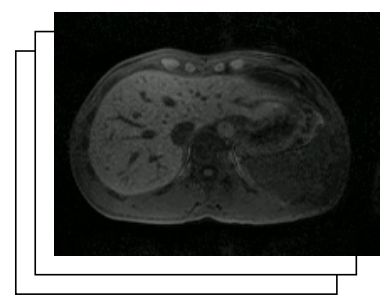

b)

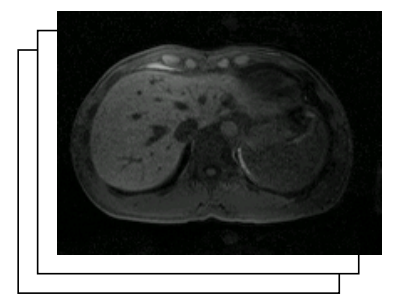

c)

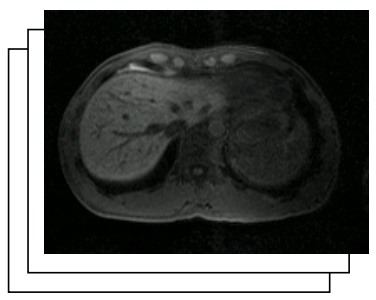

Fig. 3. a) MR scan taken at maximal inhalation. b) Intermediate scan (50\% inhalation), only needed for evaluation of the method. c) MR scan taken at maximal exhalation. a and $\mathrm{c}$ are needed as input of our method.

\subsection{Deformation by Barycentric Coordinates}

First the two volumes are tessellated into tetrahedrons by Delaunay tetrahedrization. The control points and the 8 vertices of the bounding box of the volume are used as vertices for this tetrahedrization. During deformation, the vertices are linearly interpolated over time. In each interpolation step, the barycentric coordinates are calculated in relation to the enclosing tetrahedron, for all the points of the actual stack. Using these weights the positions of the corresponding pixels of the two source images are computed. The value of the new pixel is linearly interpolated between these pixels.

\subsection{Deformation by Thin-Plate Splines}

The second approach is based on thin-plate splines, which were introduced by Duchon in 1976 [3], but in the context of analysis of plane mappings they were first considered by Bookstein [4]. A thin-plate spline interpolates a surface that is constrained not to move at given control points. This surface represents a thin metal plate, which will be deformed into the most economic shape, in reference to its bending energy. The following quantity represents the bending energy of the thin-plate function in 3D space.

$\iiint_{R^{3}}\left[\left(\frac{\partial^{2} f}{\partial x^{2}}\right)^{2}+\left(\frac{\partial^{2} f}{\partial y^{2}}\right)^{2}+\left(\frac{\partial^{2} f}{\partial z^{2}}\right)^{2}+2\left(\frac{\partial^{2} f}{\partial x \partial y}\right)^{2}+2\left(\frac{\partial^{2} f}{\partial x \partial z}\right)^{2}+2\left(\frac{\partial^{2} f}{\partial y \partial z}\right)^{2}\right] d x d y d z$ 
The thin-plate spline function is:

$$
f(x, y, z)=a_{1}+a_{x} x+a_{y} y+a_{z} z+\sum_{i=1}^{K} w_{i} U\left(\left|Z_{i}-(x, y, z)\right|\right)
$$

The function consists of an affine part and a sum of functions $U(r)$, whereas in $3 \mathrm{D}$ space $U_{T P S}(r)=|r|$ is used.

In our approach we linearly interpolate the corresponding control point lines and in each interpolation step determine the coefficients of the thin-plate spline function. The number of interpolation steps depends on the number of desired target scans. This method deforms the inhalation scan into the exhalation scan step by step, yielding the 3D motion picture, needed to infer the respiratory state.

\subsection{Results}

To evaluate the warping algorithms, MR scans of the liver and lung were used. Tests with CT scans are in process. They are obligatory for the whole application of tracking tumors, because of the DRR based registration process.

Both of the methods were tested with 91 control point pairs selected manually by an expert and the two image stacks, one inhalation, one exhalation, (3) as input. 9 intermediate scans, for later registration, were calculated. Calculation times were 64 minutes for the thin-plate spline based method and 69 minutes for the tetrahedrization based method, respectively. Both computations were performed on a $2 \mathrm{GHz}$ standard personal computer.

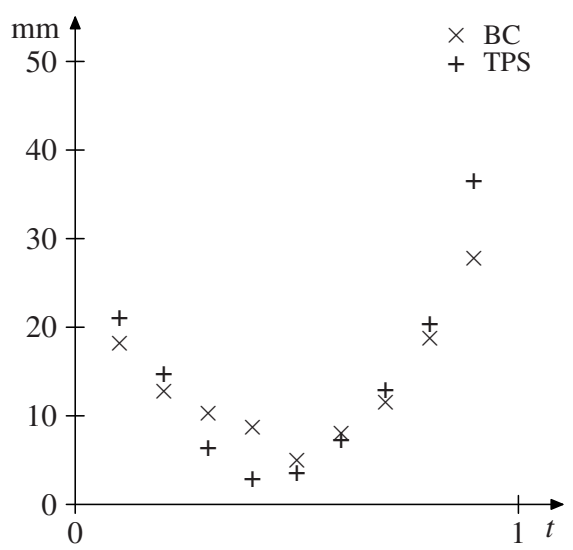

Fig. 4. The surfaces of the liver of all the synthetic intermediate scans were compared to the real intermediate scan. The best results are in the middle of the whole respiration, where the real intermediate scan is located. "TPS" stands for the thin-plate spline based method and "BC" for the tetrahedrization based method. 
To evaluate the results we compared them with an intermediate scan, located right between the two initial scans (3). For evaluation, first the liver in the two input scans and in the intermediate scan was segmented. Then the calculations were performed on these images and the same control point pairs as before. After that the reconstructed surfaces of the synthetic intermediate scans were compared to the surface of the real intermediate scan, in order to get the average deviation of the surfaces in $\mathrm{mm}$. The surfaces were reconstructed using the Marching Cubes Algorithm [11. For each of the surface triangles' vertices of the real intermediate state, the distance to the nearest point on the nearest triangle of the computed intermediate state was calculated. The average deviation is taken to be the mean value of these distances.

As expected, the intermediate data set matches the artificial 4D model best at the middle of the time period of the respiratory cycle (cf. figure 4). The tetrahedrization based method lead to an average deviation of $4.94 \mathrm{~mm}$ and the thin-plate spline based method to an average deviation of $3.82 \mathrm{~mm}$.

Additionally we generated some difference images to visualize the results. Figure 5 shows an example with the original image data, and in figure 6 with the segmented data.

b)

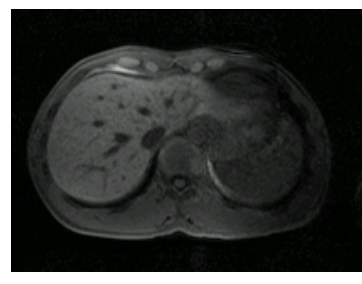

d)

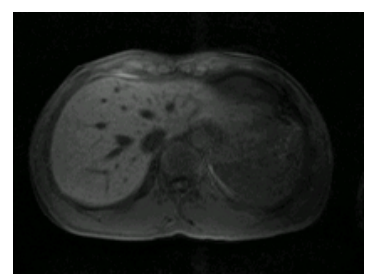

c)

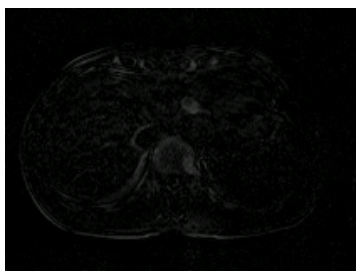

e)

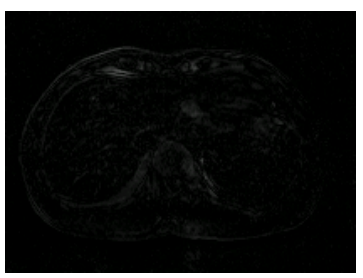

Fig. 5. a) Reference slice of the intermediate scan. b) Corresponding slice of the synthetic scan, same respiratory state, calculated with the thin-plate spline method. c) Difference between a and b. d) Corresponding slice of the synthetic scan, same respiratory state, calculated with the tetrahedrization based method. e) Difference between a and d. 
b)

a)

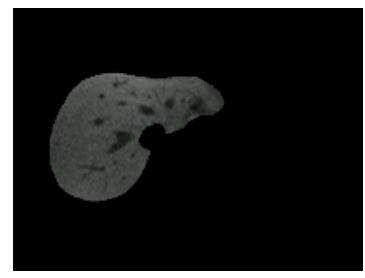

d) c)
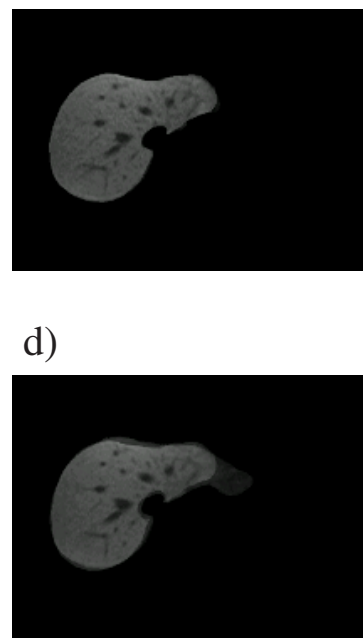

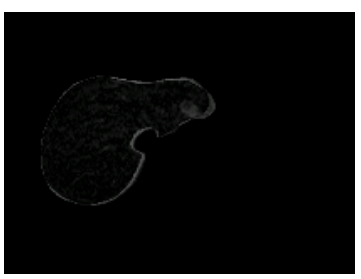

e)

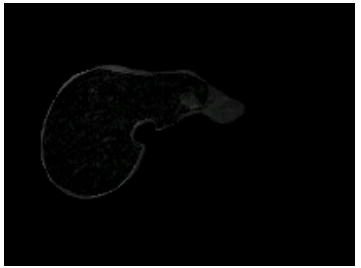

Fig. 6. a) Segmented liver of 5 a b) Segmented liver of 5 b c) Difference between a and b. d) Segmented liver of 5 d e) Difference between a and d.

\section{Conclusion and Future Work}

We have shown that the computation of the deformation of internal organs due to respiration is feasible. Starting with two data sets, one at the inhal one at the exhal position, the prediction of the target position could be significantly improved using our approach. Our next steps are: Accomplishing a cohort study with a set of intermediate respiratory status CT scans. This will help us to refine our deformation models in order to reduce the residual deviations of artificial data compared to the true trajectories of organ movements.

A procedure is developed, which helps finding control points automatically or at least semi-automatically.

The next clinical goal is to create procedures for obtaining 4D CT images similar to Pan et al. in [8]. For inferring the respiratory state we also will consider the possibility of using portal images instead of radiographs.

\section{References}

1. Schweikard, A., Glosser, G., Bodduluri, M., Murphy, M., Adler, J. R., Robotic Motion Compensation for Respiratory Movement during Radiosurgery. ComputerAided Surgery, Sept. 2000

2. Roth, M., Dötter, M., Burgkart, R., Schweikard, A., Fast intensity-based fluoroscopy-to-CT registration using pattern search optimization, 18th Int. Congress Computer Assisted Radiology and Surgery (CARS 2004), 2004

3. Duchon, J., Interpolation des fonctions de deux variables suivant le principe de la flexion des plaques minces. RAIRO Analyse Numérique, 1976 
4. Bookstein, F. L., Principal warps: thin-plate splines and the decomposition of deformations. IEEE Transactions on Pattern Analysis an Machine Intelligence, 1989, 11:567-85

5. Schweikard, A., Shiomi, H., Dötter, M., Roth, M., Berlinger, K., Adler, J. R., Fiducial-less compensation of breathing motion in lung cancer radiosurgery. Technical Report A-02-23, Informatik, Universität Lübeck, Germany, 2002

6. Fornefett, M., Rohr, K., Stiehl, H. S., Radial Basis Functions with Compact Support for Elastic Registration of Medical Images. Workshop on Biomedical Image Registration, Aug. 1999, 173-185

7. Kubo, H. D., Hill, B. C., Respiration gated radiotherapy treatment: a technical study. Phys. Med. Biol., 1996, 41: 83-91

8. Pan, T., Lee, T.-Y., Rietzel, E., Chen, G. T. Y., 4D-CT imaging of a volume influenced by respiratory motion on multi-slice CT. Med. Phys., Feb 2004, Vol. 31 No. 2 333-340

9. Brock, K. K., Hollister, S. J., Dawson, L. A., Balter, J. M., Technical note: Creating a four-dimensional model of the liver using finite element analysis. Med. Phys., July 2002, Vol. 29 No. 7 1403-1405

10. Salomon, D., Computer Graphics and Geometric Modeling. Springer-Verlag New York, Inc. ISBN 0-387-98682-0

11. Lorensen, W. E., Cline, H. E., Marching cubes: A high resolution 3D surface construction algorithm. International Conference on Computer Graphics and Interactive Techniques, 1987, 163-169 